Supporting Information for

\title{
The Regioselectivity of Pyridine Deprotonation in the Gas Phase
}

\author{
By \\ Bonnie S. Schafman and Paul G. Wenthold
}

\section{Contents:}

S2-S3: Description of the determination of the gas-phase acidity of pyridine taking into account the formation of a mixture of isomers

\section{S4-S11: Figures S1 - S9}

Branching ratios, representative plots, and $2^{\text {nd }}$ regression plots for the determination of the acidity of pyridine carboxylic acids by using the kinetic method

Full references: S13

S14-S21: Z-matrices and Calculated Energies: 


\section{Determination of the acidity of pyridine:}

Meot-ner and Kafafi (MK)

J. Am. Chem. Soc. 1988, 110, 6297

MK report the following results from temperature dependent equilibrium measurements

$\mathrm{OH}^{-}+\mathrm{C}_{5} \mathrm{H}_{5} \mathrm{~N}=\mathrm{H}_{2} \mathrm{O}+\mathrm{C}_{5} \mathrm{H}_{4} \mathrm{~N}^{-}$

$\Delta \mathrm{H}^{\circ}=1.1 \mathrm{kcal} / \mathrm{mol}$

$\Delta \mathrm{S}^{\circ}=9.8 \mathrm{eu}$

$\mathrm{CH}_{3} \mathrm{O}^{-}+\mathrm{C}_{5} \mathrm{H}_{5} \mathrm{~N}=\mathrm{CH}_{3} \mathrm{OH}+\mathrm{C}_{5} \mathrm{H}_{4} \mathrm{~N}^{-}$

$\Delta \mathrm{H}^{\circ}=8.5 \mathrm{kcal} / \mathrm{mol}$

$\Delta \mathrm{S}^{\circ}=6.7 \mathrm{eu}$

From these, we conclude that $\delta \Delta \mathrm{G}$ for the reactions at room temp are

$-1.8 \mathrm{kcal} / \mathrm{mol}$ for the reaction with $\mathrm{OH}^{-}$

$+6.5 \mathrm{kcal} / \mathrm{mol}$ for $\mathrm{CH}_{3} \mathrm{O}^{-}$

where $\delta \Delta \mathrm{G}^{\circ}{ }_{\text {acid }}=\Delta \mathrm{G}^{\circ}{ }_{\text {acid }}($ pyridine $)-\Delta \mathrm{G}^{\circ}{ }_{\text {acid }}($ reference $)$.

By using the acidities of water $(383.7 \mathrm{kcal} / \mathrm{mol})$ and methanol $(375.5 \pm 0.6 \mathrm{kcal} / \mathrm{mol})$ from the recent paper by DeTuri and Ervin (J. Phys. Chem. A, 2002, 106, 9947), we obtain values of 381.9 and $382.0 \mathrm{kcal} / \mathrm{mol}$ for $\Delta \mathrm{G}_{\text {acid }}$ (pyridine). When using the value of $\Delta \mathrm{S}^{\circ}$ acid $=27.6 \mathrm{cal} / \mathrm{mol} \mathrm{K}$, recommended by $\mathrm{MK}$, we obtain $\Delta \mathrm{H}^{\circ}$ acid $($ pyridine $)=390.2$ $\mathrm{kcal} / \mathrm{mol}$.

However, the $\mathrm{C}_{5} \mathrm{H}_{4} \mathrm{~N}^{-}$ions formed in the deprotonation of pyridine are a mixture of meta and para isomers. We estimate that the composition is $70-80 \%$ para, and the rest is meta. That has an effect on the equilibrium measurement, because there is more than one product. We can view the problem as the following

$\mathrm{OH}^{-}+\mathrm{C}_{5} \mathrm{H}_{5} \mathrm{~N}=\mathrm{H}_{2} \mathrm{O}+\mathrm{C}_{5} \mathrm{H}_{4} \mathrm{~N}^{-}$

$\mathrm{K}_{\text {meas }}=\left[\mathrm{C}_{5} \mathrm{H}_{4} \mathrm{~N}^{-}\right]\left[\mathrm{H}_{2} \mathrm{O}\right] /\left[\mathrm{OH}^{-}\right]\left[\mathrm{C}_{5} \mathrm{H}_{5} \mathrm{~N}\right]$

However, $\left[\mathrm{C}_{5} \mathrm{H}_{4} \mathrm{~N}^{-}\right]=[$meta $]+[$para $]$, so the expression becomes

$$
\mathrm{K}_{\text {meas }}=\frac{([\text { meta }]+[\text { para }])\left[\mathrm{H}_{2} \mathrm{O}\right]}{\left[\mathrm{OH}^{-}\right][\text {pyridine }]}=\frac{[\text { meta }]\left[\mathrm{H}_{2} \mathrm{O}\right]}{\left[\mathrm{OH}^{-}\right][\text {pyridine }]}+\frac{[\text { para }]\left[\mathrm{H}_{2} \mathrm{O}\right]}{\left[\mathrm{OH}^{-}\right][\text {pyridine }]}=\mathrm{K}_{\text {meta }}+\mathrm{K}_{\text {para }}
$$


From this expression, we can see that

$\mathrm{K}_{\text {para }} / \mathrm{K}_{\text {meas }}=[$ para $] /([$ meta $]+[$ para $])=0.7-0.8$

Therefore, the equilibrium constant for formation of the para anion is $0.7-0.8$ of the measured $\mathrm{K}$.

At room temperature, the $\mathrm{K}_{\text {meas }}$ value for the reaction with water $(\Delta \mathrm{G}=-1.8)$ is 20.5 . This means that $\mathrm{K}_{\text {para }}=14.3-16.4$. Similarly, the $\mathrm{K}_{\text {meas }}$ for the reaction with methanol is $1.87 \times 10^{-5}$, giving $\mathrm{K}_{\text {para }}=1.30-1.50 \times 10^{-5}$. Therefore the $\Delta \mathrm{G}$ values for formation of the para anion are

Water: $-1.6--1.7 \mathrm{kcal} / \mathrm{mol}$

Methanol: $6.7-6.6 \mathrm{kcal} / \mathrm{mol}$

When using water as a reference, we calculate the acidity at the para position to be 382.0 - 382.1 kcal/mol, and using methanol, we obtain 382.1 - $382.2 \mathrm{kcal} / \mathrm{mol}$. Thus, we assign the acidity at the para position in pyridine to be $382.1 \mathrm{kcal} / \mathrm{mol}$. We have retained the $\pm 2.0 \mathrm{kcal} / \mathrm{mol}$ uncertainty assigned by $\mathrm{MK}$. The meta position is measured to be 0.9 $-1.2 \mathrm{kcal} / \mathrm{mol}$ less acidic, giving a value of $\mathbf{3 8 3 . 0}-\mathbf{3 8 3 . 3} \mathrm{kcal} / \mathrm{mol}$. 
Figure S1. Energy-Resolved Branching Ratios for Dissociation of Proton-Bound Dimers Formed from Deprotonated Picolinic Acid

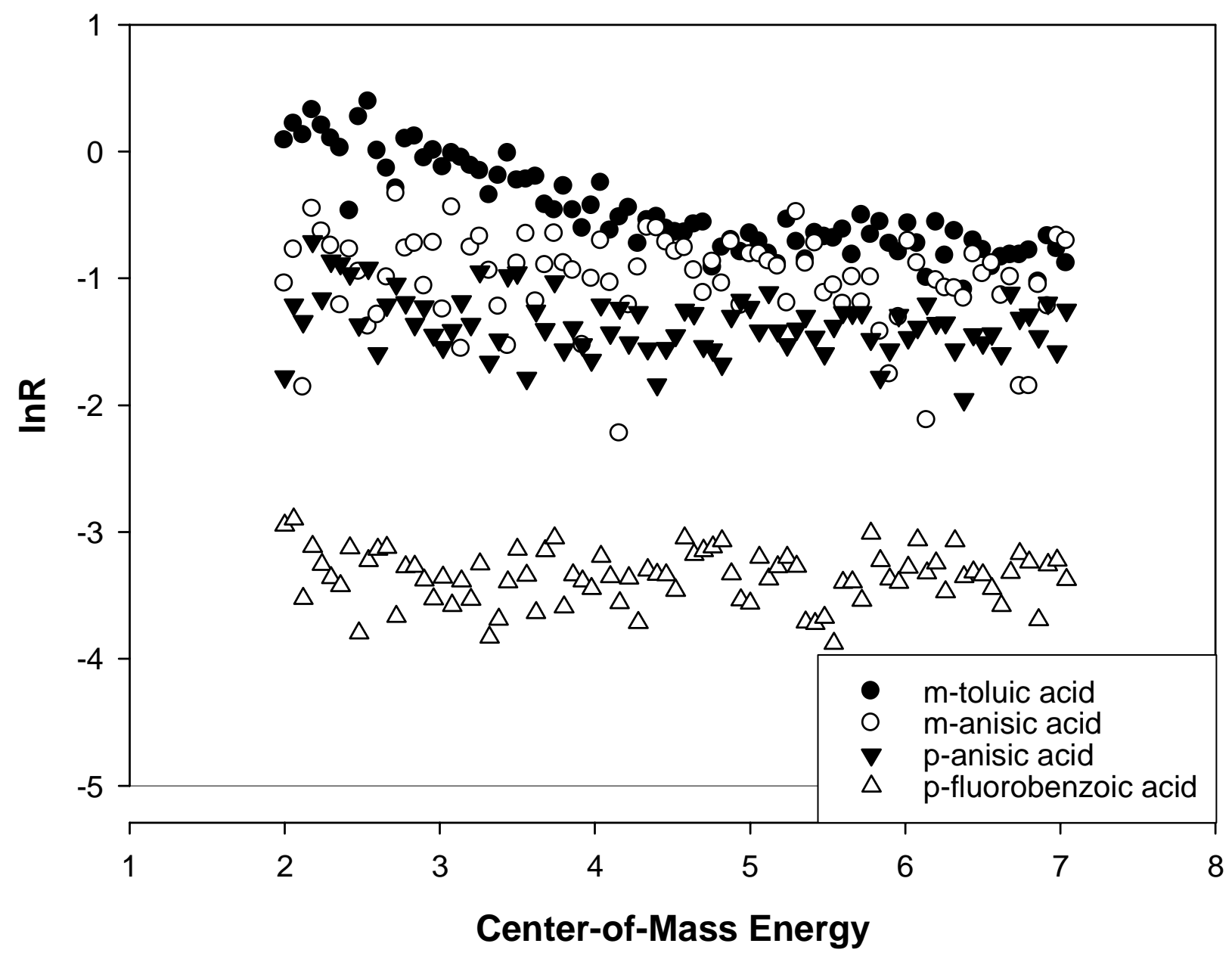


Figure S2. Plots of $\operatorname{lnR}$ vs $\left(\Delta \mathrm{H}^{\circ}\right.$ acid $-\Delta \mathrm{H}^{\circ}$ avg $)$ for the References shown in Figure $\mathrm{S} 1$ at Representative Energies. For these References, $\Delta \mathrm{H}^{\circ}$ avg $=339.2 \pm 1.4$ $\mathrm{kcal} / \mathrm{mol}$

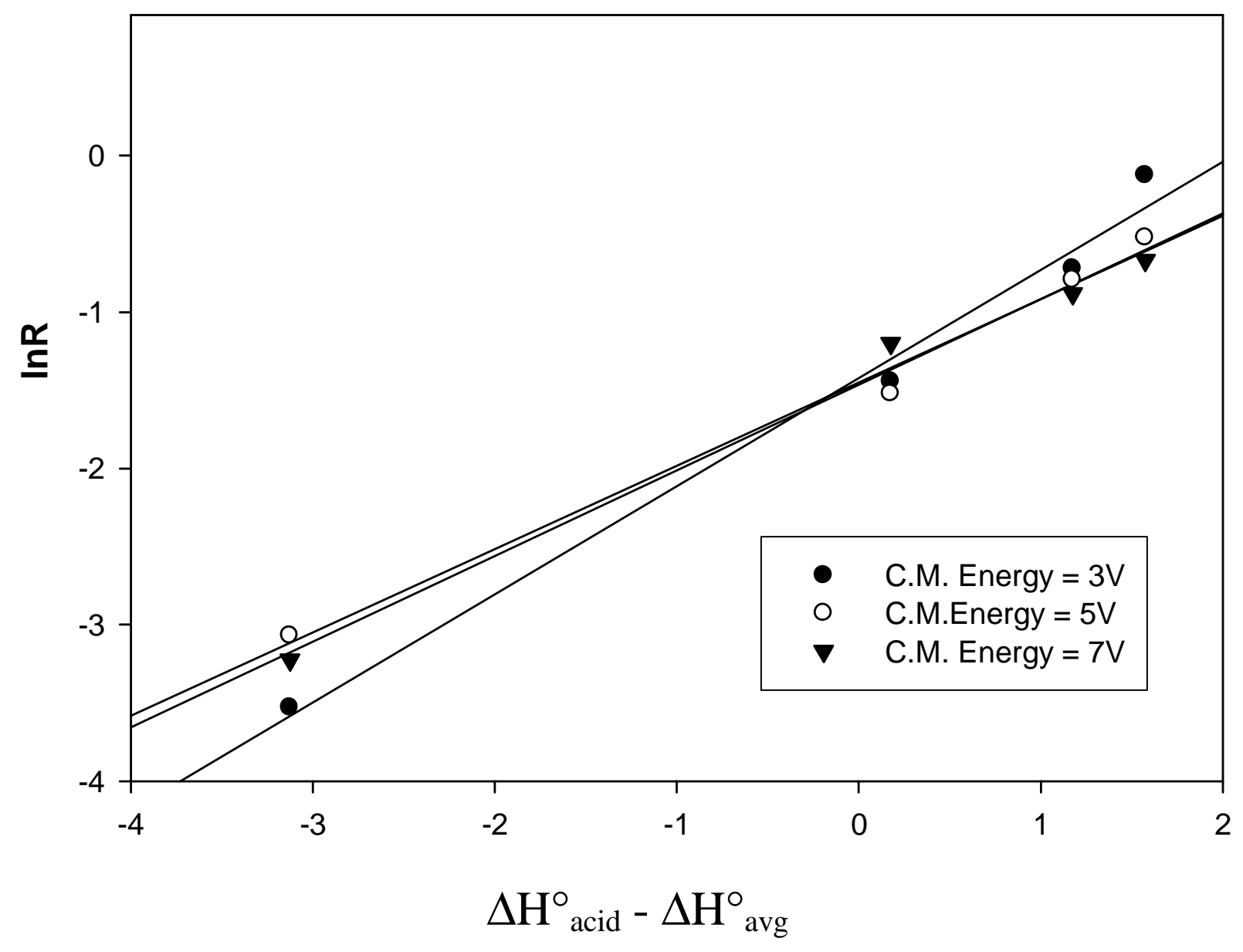


Figure S3. Second Regression Plot for the Determination of the Gas Phase Acidity of Picolinic Acid. Combination of the Slope with $\Delta \mathrm{H}^{\circ}{ }_{\text {avg }}$ from Figure $\mathrm{S} 2$ gives $\Delta \mathrm{H}^{\circ}{ }_{\text {acid }}=343.9 \pm 1.8 \mathrm{kcal} / \mathrm{mol}$

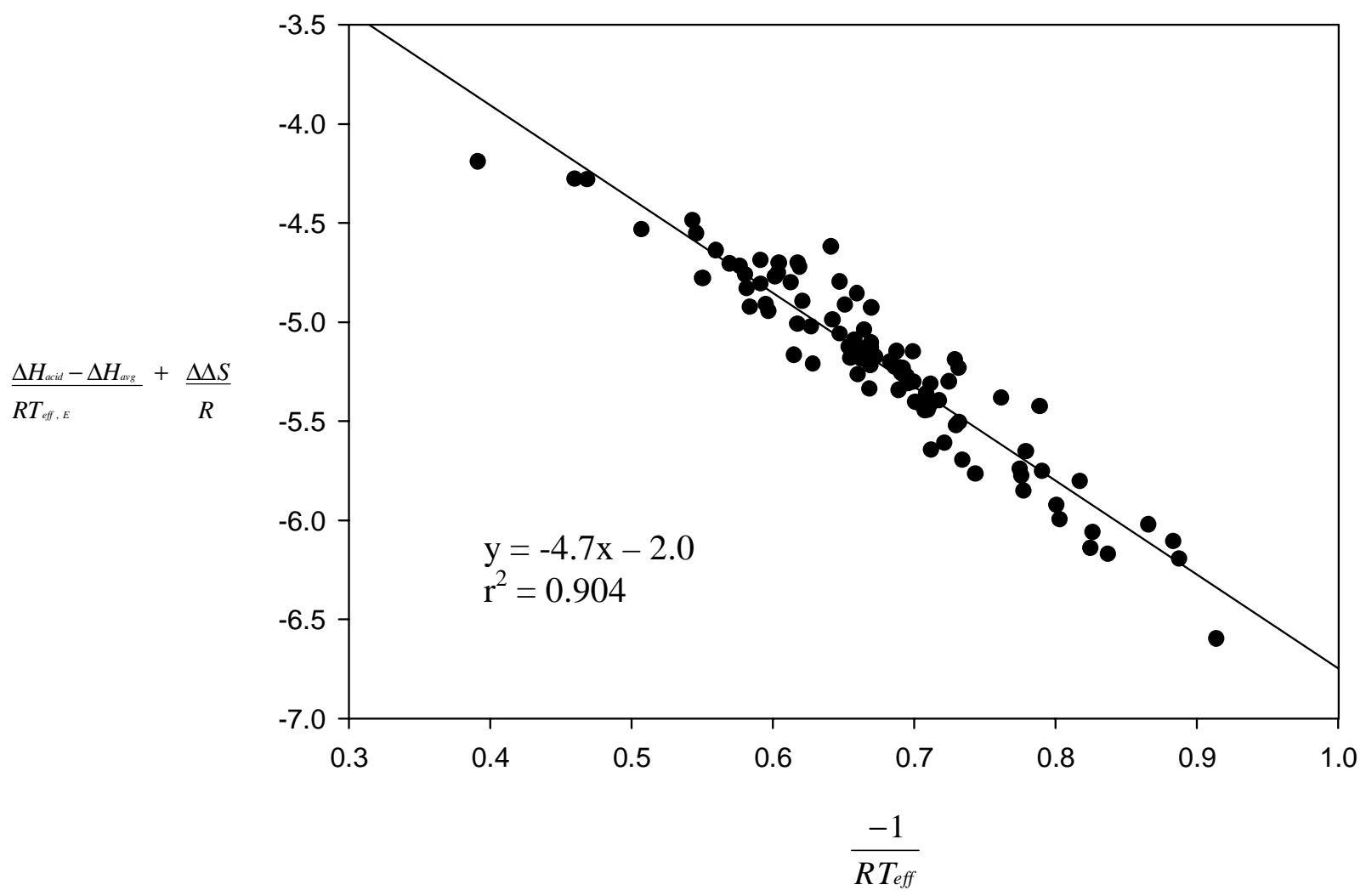


Figure S4. Energy-Resolved Branching Ratios for Dissociation of Proton-Bound Dimers Formed from Deprotonated Isonicotinic Acid

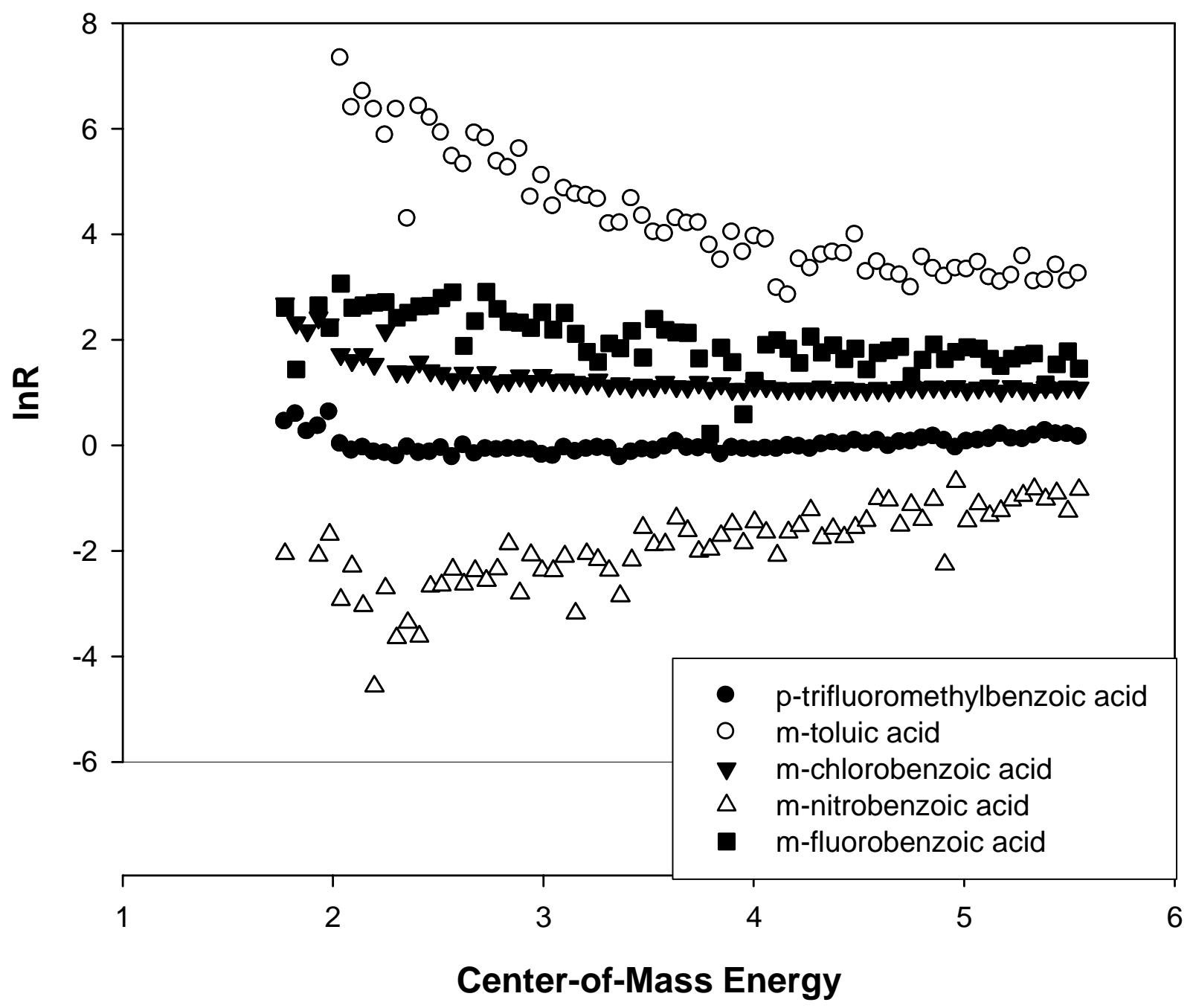


Figure S5. Plots of $\operatorname{lnR}$ vs $\left(\Delta \mathrm{H}^{\circ}{ }_{\text {acid }}-\Delta \mathrm{H}^{\circ}{ }_{\text {avg }}\right)$ for the References shown in Figure $\mathrm{S} 4$ at Representative Energies. For these References, $\Delta \mathrm{H}^{\circ}{ }_{\text {avg }}=342.3 \pm 1.4$ $\mathrm{kcal} / \mathrm{mol}$

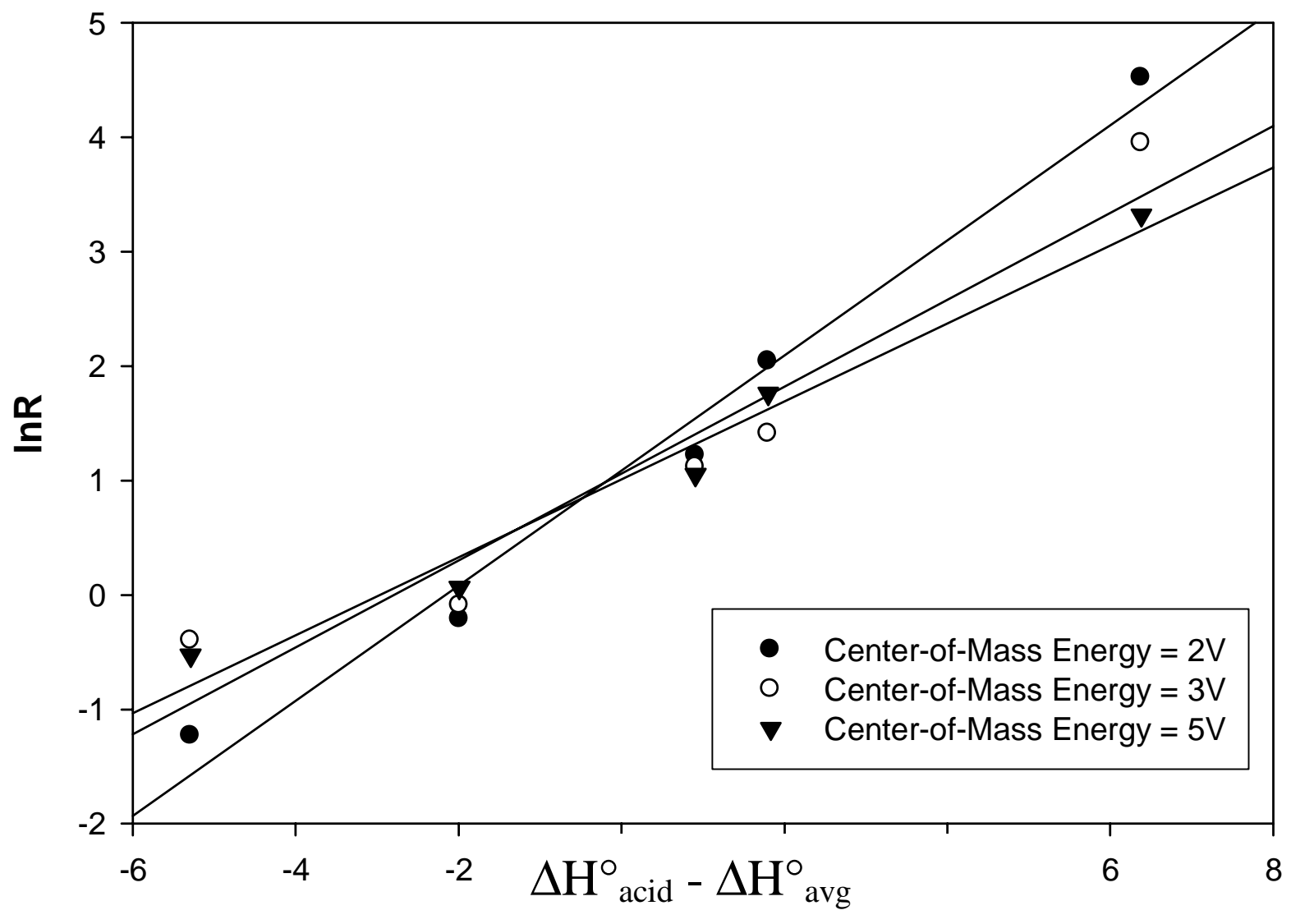


Figure S6. Second Regression Plot for the Determination of the Gas Phase Acidity of Isonicotinic Acid. Combination of the Slope with $\Delta \mathrm{H}^{\circ}{ }_{\text {avg }}$ from Figure $\mathrm{S} 5$ gives $\Delta \mathrm{H}^{\circ}{ }_{\text {acid }}=332.6 \pm 1.7 \mathrm{kcal} / \mathrm{mol}$

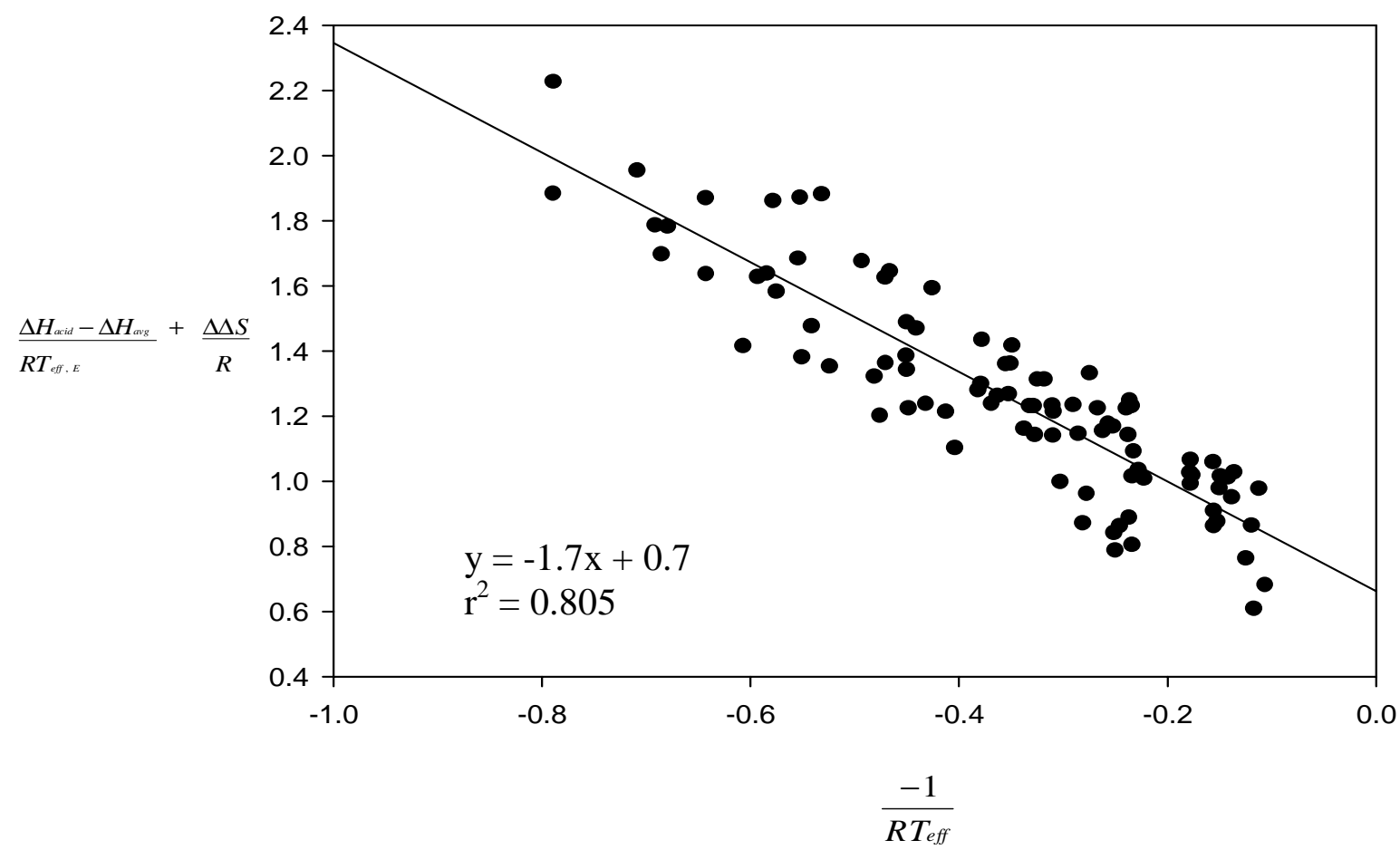


Figure S7. Energy-Resolved Branching Ratios for Dissociation of Proton-Bound Dimers Formed from Deprotonated Nicotinic Acid

InR vs. Energy

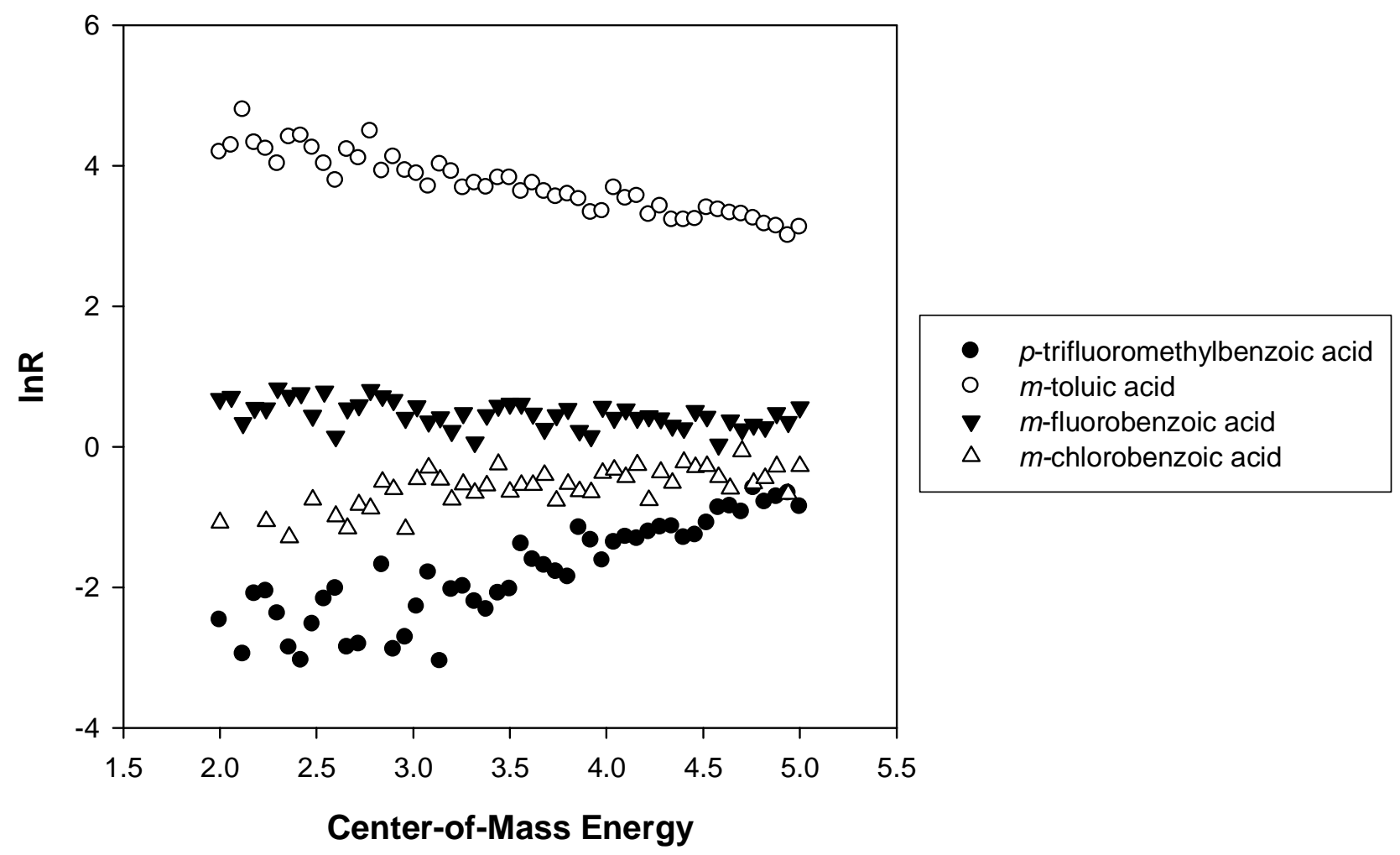


Figure S8. Plots of $\ln \mathrm{R} v \mathrm{vs}\left(\Delta \mathrm{H}^{\circ}\right.$ acid $-\Delta \mathrm{H}^{\circ}$ avg $)$ for the References shown in Figure $\mathrm{S} 7$ at Representative Energies. For these References, $\Delta \mathrm{H}^{\circ}{ }_{\text {avg }}=334.6 \pm 1.4$ $\mathrm{kcal} / \mathrm{mol}$

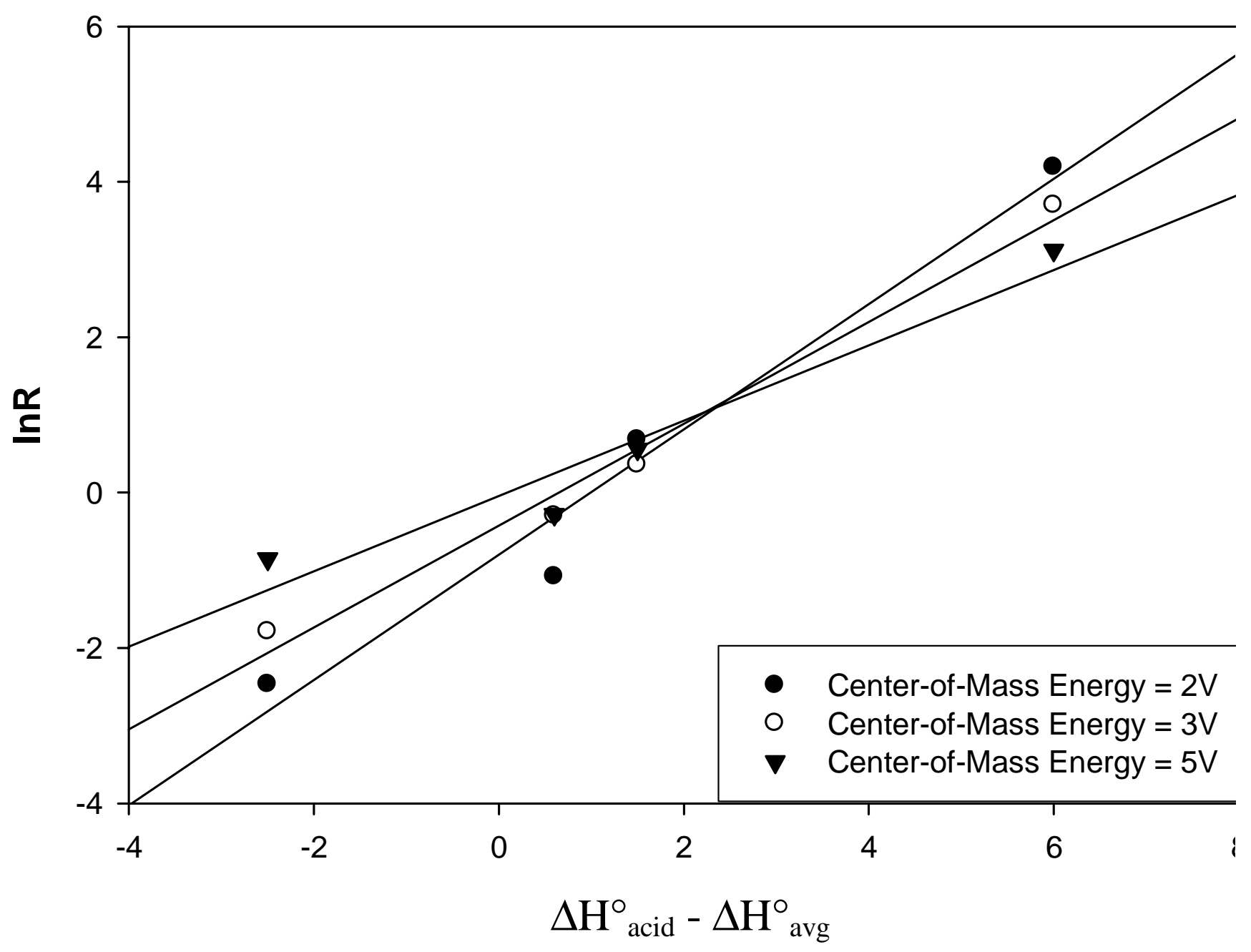


Figure S9. Second Regression Plot for the Determination of the Gas Phase Acidity of Nicotinic Acid. Combination of the Slope with $\Delta \mathrm{H}_{\mathrm{avg}}$ from Figure $\mathrm{S} 8$ gives $\Delta \mathrm{H}_{\mathrm{acid}}=334.4 \pm 2.0 \mathrm{kcal} / \mathrm{mol}$

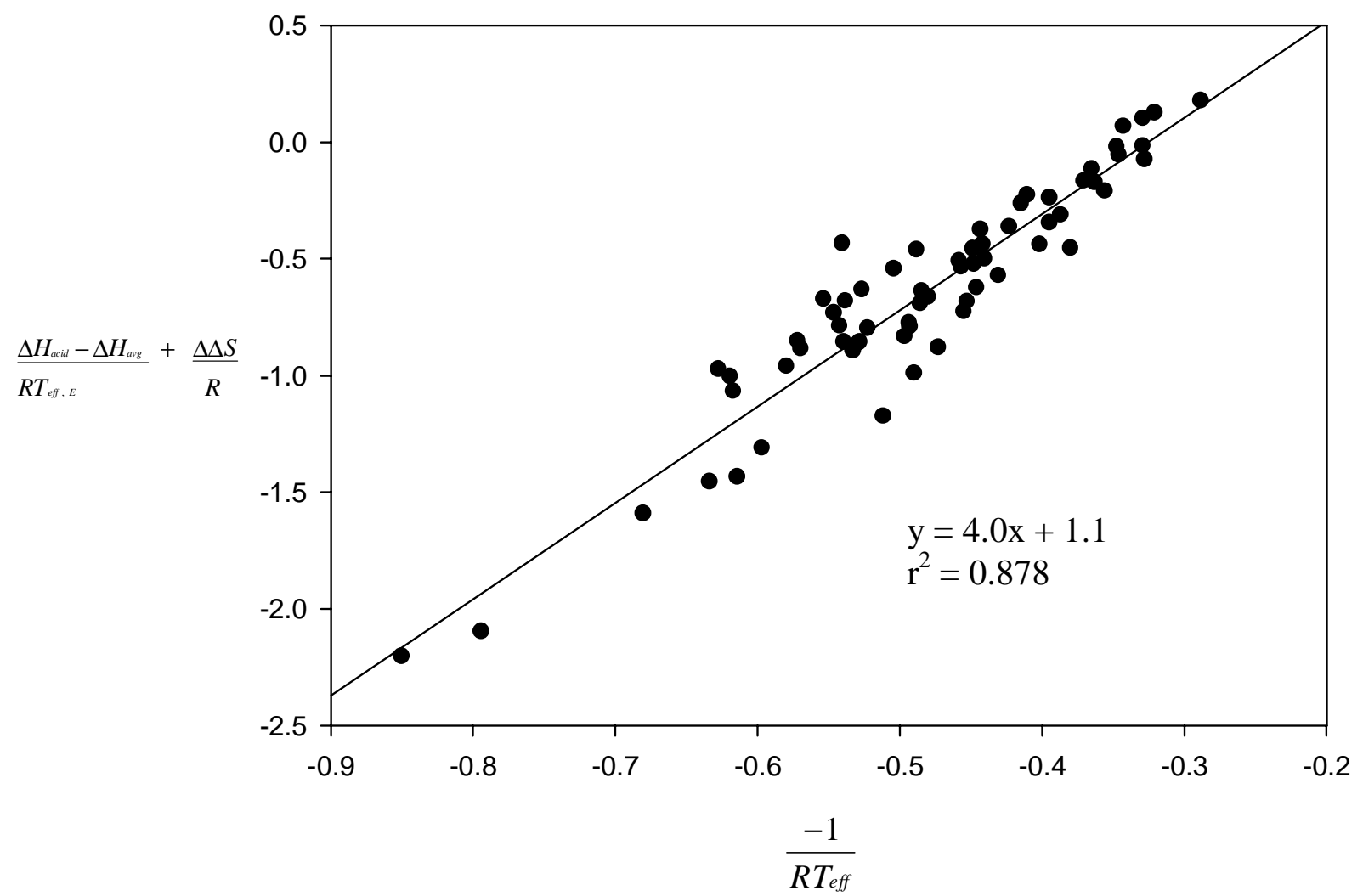




\section{Full References:}

(33) M. J. Frisch; G. W. Trucks; H. B. Schlegel; G. E. Scuseria; M. A. Robb; J. R. Cheeseman; J. Montgomery, J. A.; T. Vreven; K. N. Kudin; J. C. Burant; J. M. Millam; S. S. Lyengar; J. Tomasi; V. Barone; B. Mennucci; M. Cossi; G. Scalmani; N. Rega; G. A. Petersson; H. Nakatsuji; M. Hada; M. Ehara; K. Toyota; R. Fukuda; J. Hasegawa; M. Ishida; T. Nakajima; Y. Honda; O. Kitao; H. Nakai; M. Klene; X. Li; J. E. Knox; H. P. Hratchian; J. B. Cross; V. Bakken; C. Adamo; J. Jaramillo; R. Gomperts; R. E. Stratmann; O. Yazyev; A. J. Austin; R. Cammi; C. Pomelli; J. W. Ochterski; P. Y. Ayala; K. Morokuma; G. A. Voth; P. Salvador; J. J. Dannenberg; V. G. Zakrzewski; S. Dapprich; A. D. Daniels; M. C. Strain; O. Farkas; D. K. Malick; A. D. Rabuck; K. Raghavachari; J. B. Foresman; J. V. Ortiz; Q. Cui; A. G. Baboul; S. Clifford; J. Cioslowski; B. B. Stefanov; G. Liu; A. Liashenko; P. Piskorz; I. Komaromi; R. L. Martin; D. J. Fox; T. Keith; M. A. Al-Laham; C. Y. Peng; A. Nanayakkara; M. Challacombe; P. M. W. Gill; B. Johnson; W. Chen; M. W. Wong; C. Gonzalez; J. A. Pople, Gaussian 03, Revision B.04; Gaussian, Inc.; Pittsburgh, PA, 2003

(64) Frisch, M. J.; Trucks, G. W.; Schlegel, H. B.; Scuseria, G. E.; Robb, M. A.; Cheeseman, J. R.; Zakrzewski, V. G.; J. A. Montgomery, J.; Stratmann, R. E.; Burant, J. C.; Dapprich, S.; Millam, J. M.; Daniels, A. D.; Kudin, K. N.; Strain, M. C.; Farkas, O.; Tomasi, J.; Barone, V.; Cossi, M.; Cammi, R.; Mennucci, B.; Pomelli, C.; Adamo, C.; Clifford, S.; Ochterski, J.; Petersson, G. A.; Ayala, P. Y.; Cui, Q.; Morokuma, K.; Malick, D. K.; Rabuck, A. D.; Raghavachari, K.; Foresman, J. B.; Cioslowski, J.; Ortiz, J. V.; Baboul, A. G.; Stefanov, B. B.; Liu, G.; Liashenko, A.; Piskorz, P.; Komaromi, I.; Gomperts, R.; Martin, R. L.; Fox, D. J.; Keith, T.; Al-Laham, M. A.; Peng, C. Y.; Nanayakkara, A.; Gonzalez, C.; Challacombe, M.; Gill, P. M. W.; Johnson, B. G.; Chen, W.; Wong, M. W.; Andres, J. L.; Head-Gordon, M.; Replogle, E. S.; Pople, J. A.Gaussian 98, Revision A.09; Gaussian, Inc.; Pittsburgh, PA, 1998 


\section{Z-matrices and Calculated Energies}

\section{Ortho anion:}

\#p b3lyp/aug-cc-pvdz guess=check geom=check opt freq symm=loose

Charge $=-1$ Multiplicity $=1$

$\mathrm{C}, 0,-1.2688862022,0 .,-0.8312986554$

C, $0,-1.2353051536,0 ., 0.604070333$

$\mathrm{C}, 0,-0.0616962473,0 ., 1.3607359854$

C, $0,1.1643521352,0 ., 0.6814066353$

C, $0,1.1100332507,0 .,-0.7160446748$

$\mathrm{N}, 0,-0.0204218977,0 .,-1.4444402519$

$\mathrm{H}, 0,-0.0891755559,0 ., 2.4590278104$

$\mathrm{H}, 0,-2.1950626416,0 ., 1.1378345984$

$\mathrm{H}, 0,2.123864885,0 ., 1.2068193226$

H,0,2.0523398993,0.,-1.2858177087

$\mathrm{HF}=-247.664716$

\#p mp2/aug-cc-pvdz symm=loose guess=check geom=check

Charge $=-1$ Multiplicity $=1$

C,0,-1.2688862022,0.,-0.8312986554

C, $0,-1.2353051536,0,0.604070333$

C, $0,-0.0616962473,0 ., 1.3607359854$

C, $0,1.1643521352,0,0.6814066353$

C, $0,1.1100332507,0 .,-0.7160446748$

$\mathrm{N}, 0,-0.0204218977,0 .,-1.4444402519$

$\mathrm{H}, 0,-0.0891755559,0 ., 2.4590278104$

$\mathrm{H}, 0,-2.1950626416,0 ., 1.1378345984$

$\mathrm{H}, 0,2.123864885,0 ., 1.2068193226$

H,0,2.0523398993,0.,-1.2858177087

$\mathrm{HF}=-246.0812812$

\section{Meta anion}

\#p b31yp/aug-cc-pvdz guess=check geom=check opt freq symm=loose

Charge $=-1$ Multiplicity $=1$

C, $0,-1.2179769845,0 .,-0.5904611613$

$\mathrm{C}, 0,-1.2798265047,0 ., 0.8301385807$

C, $0,0.0230797881,0 ., 1.3902455893$

C,0,1.1942433961,0.,0.6136395831 
C, $0,1.0688672272,0 .,-0.7778137171$

$\mathrm{N}, 0,-0.1243180084,0 .,-1.3946292497$

$\mathrm{H}, 0,0.1478786837,0 ., 2.4852773625$

$\mathrm{H}, 0,-2.157184459,0 .,-1.1653856131$

$\mathrm{H}, 0,2.1924203853,0 ., 1.0704431777$

$\mathrm{H}, 0,1.9567899148,0 .,-1.4224234273$

$\mathrm{HF}=-247.6775241$

\#p mp2/aug-cc-pvdz guess=check geom=check symm=loose opt

Charge $=-1$ Multiplicity $=1$

C, $0,-1.2176420319,0 .,-0.5905359039$

C, $, 0,-1.279792421,0 ., 0.8301909769$

C, $0,0.0229058929,0 ., 1.3903964578$

C, $0,1.1942870088,0 ., 0.6137109237$

C, $0,1.0686276596,0 .,-0.7782140544$

$\mathrm{N}, 0,-0.1243198431,0 .,-1.394520154$

$\mathrm{H}, 0,0.1473918283,0 ., 2.4853979404$

$\mathrm{H}, 0,-2.1569862109,0 .,-1.16499958$

$\mathrm{H}, 0,2.1927221545,0 ., 1.0704580582$

$\mathrm{H}, 0,1.9567944792,0 .,-1.4225057415$

$\mathrm{HF}=-246.1220018$

\section{Para Anion:}

\#p opt guess=check geom=check b3lyp/aug-cc-pVDZ symm=loose

Charge $=-1$ Multiplicity $=1$

$\mathrm{C}, 0,-1.1518679065,0.000000001,-0.6579500122$

C, $0,-1.1648317737,0.0000000003,0.7371760045$

C, $0,0.0178774493,-0.0000000008,1.5510313408$

C, $0,1.181513686,-0.0000000011,0.7101316303$

C, $0,1.1363966187,-0.0000000004,-0.6843249357$

$\mathrm{N}, 0,-0.0161690248,0.0000000007,-1.4028099816$

$\mathrm{H}, 0,-2.0893136429,0.0000000019,-1.2246872536$

$\mathrm{H}, 0,-2.1532243412,0.0000000007,1.2152225625$

$\mathrm{H}, 0,2.1806622943,-0.0000000019,1.165269453$

$\mathrm{H}, 0,2.0605304211,-0.0000000006,-1.2725190573$

$\mathrm{HF}=-247.6808053$ 
\#p opt mp2/aug-cc-pvdz symm=loose guess=check geom=check

Charge $=-1$ Multiplicity $=1$

C, $0,-1.1404815966,0.000000001,-0.6593765595$

C, $, 0,-1.1574309787,0.0000000003,0.7410144943$

C, $0,0.0177971761,-0.0000000008,1.5440669129$

C, $0,1.1742033316,-0.0000000011,0.7141396834$

C, $0,1.124980453,-0.0000000003,-0.6854886578$

$\mathrm{N}, 0,-0.0161433807,0.0000000007,-1.4005851208$

$\mathrm{H}, 0,-2.0831009485,0.0000000019,-1.2290245103$

$\mathrm{H}, 0,-2.1513462177,0.0000000007,1.2168036601$

$\mathrm{H}, 0,2.178821113,-0.0000000019,1.16689342$

H,0,2.0542194066,-0.0000000006,-1.2767119636

$\mathrm{HF}=-246.1281757$

\section{Nicotinic acid:}

\#p b3lyp/aug-cc-pvdz opt freq guess=check geom=check symm=loose

Charge $=0$ Multiplicity $=1$

C, $0,-2.2020878292,0 .,-1.2248414797$

C, $0,-2.2065074103,0 ., 0.1731821952$

C, $0,-0.9852866376,0,0.8426197978$

C, $0,0.1917205591,0 ., 0.0830420126$

C, $0,0.0829714504,0 .,-1.3169533521$

$\mathrm{N}, 0,-1.0846242389,0,-1.9665853183$

$\mathrm{H}, 0,-3.1392547244,0 .,-1.7782997242$

$\mathrm{H}, 0,-3.1454924229,0,0.718994315$

$\mathrm{H}, 0,0.9842351511,0 .,-1.9242896227$

$\mathrm{H}, 0,-0.9383564656,0 ., 1.9265812926$

C, $0,1.5462910016,0 ., 0.6918252079$

$\mathrm{O}, 0,1.5125157959,0 ., 2.0512647067$

O,0,2.5911581932,0.,0.0705109539

H,0,2.4392394176,0.,2.3556593918

$\mathrm{HF}=-436.9219374$

\section{Nicotinoate:}

Charge $=-1$ Multiplicity $=1$

C,0,-2.1762182184,0.,-1.1999385241

C, $0,-2.1796966246,0 ., 0.1969461595$

C, $0,-0.9535046446,0 ., 0.8670156179$

C,0,0.2341283515,0.,0.1295882594 
C, $0,0.114216172,0 .,-1.2684661134$

$\mathrm{N}, 0,-1.0541821007,0 .,-1.9363145041$

$\mathrm{H}, 0,-3.1145437808,0 .,-1.756835868$

$\mathrm{H}, 0,-3.123276475,0 ., 0.7408952507$

$\mathrm{H}, 0,1.0229167979,0 .,-1.8646681059$

$\mathrm{H}, 0,-0.8805166985,0 ., 1.9514799855$

C, $0,1.6182441906,0 ., 0.8288055899$

$\mathrm{O}, 0,1.5793302348,0 ., 2.0876116721$

$\mathrm{O}, 0,2.612129703,0 ., 0.0573413694$

$\mathrm{HF}=-436.3784646$

\section{Nitrobenzene charge calculations:}

\# opt b3lyp/6-31+g(d) geom=connectivity pop=npa scf=tight aim

Symbolic Z-matrix:

Charge $=0$ Multiplicity $=1$

C

C $\quad 1 \quad$ B1

$\begin{array}{lllll}\mathrm{C} & 2 & \mathrm{~B} 2 & 1 & \mathrm{~A} 1\end{array}$

$\begin{array}{llllllll}\mathrm{C} & 3 & \mathrm{~B} 3 & 2 & \mathrm{~A} 2 & 1 & \mathrm{D} 1 & 0\end{array}$

$\begin{array}{llllllll}\mathrm{C} & 4 & \mathrm{~B} 4 & 3 & \mathrm{~A} 3 & 2 & \mathrm{D} 2 & 0\end{array}$

$\begin{array}{llllllll}\mathrm{C} & 1 & \mathrm{~B} 5 & 2 & \mathrm{~A} 4 & 3 & \mathrm{D} 3 & 0\end{array}$

$\begin{array}{llllllll}\mathrm{H} & 1 & \mathrm{~B} 6 & 6 & \mathrm{~A} 5 & 5 & \mathrm{D} 4 & 0\end{array}$

$\begin{array}{llllllll}\mathrm{H} & 2 & \mathrm{~B} 7 & 1 & \mathrm{~A} 6 & 6 & \mathrm{D} 5 & 0\end{array}$

$\begin{array}{llllllll}\mathrm{H} & 4 & \mathrm{~B} 8 & 3 & \mathrm{~A} 7 & 2 & \mathrm{D} 6 & 0\end{array}$

$\begin{array}{llllllll}\mathrm{H} & 5 & \mathrm{~B} 9 & 4 & \mathrm{~A} 8 & 3 & \mathrm{D} 7 & 0\end{array}$

$\begin{array}{llllllll}\mathrm{H} & 6 & \mathrm{~B} 10 & 1 & \mathrm{~A} 9 & 2 & \mathrm{D} 8 & 0\end{array}$

$\begin{array}{llllllll}\mathrm{N} & 3 & \mathrm{~B} 11 & 2 & \mathrm{~A} 10 & 1 & \mathrm{D} 9 & 0\end{array}$

$\begin{array}{llllllll}\mathrm{O} & 12 & \mathrm{~B} 12 & 3 & \mathrm{~A} 11 & 2 & \mathrm{D} 10 & 0\end{array}$

$\begin{array}{llllllll}\text { O } & 12 & \text { B13 } & 3 & \text { A12 } & 2 & \text { D11 } & 0\end{array}$

Variables:

$\begin{array}{ll}\text { B1 } & 1.39516 \\ \text { B2 } & 1.39471 \\ \text { B3 } & 1.39543 \\ \text { B4 } & 1.39483 \\ \text { B5 } & 1.39483 \\ \text { B6 } & 1.09961 \\ \text { B7 } & 1.09966 \\ \text { B8 } & 1.09968 \\ \text { B9 } & 1.09976 \\ \text { B10 } & 1.0996 \\ \text { B11 } & 1.47 \\ \text { B12 } & 1.19934\end{array}$




$\begin{array}{lc}\text { B13 } & 1.19934 \\ \text { A1 } & 120.00863 \\ \text { A2 } & 119.99416 \\ \text { A3 } & 119.99399 \\ \text { A4 } & 119.99846 \\ \text { A5 } & 120.00432 \\ \text { A6 } & 119.98077 \\ \text { A7 } & 119.98114 \\ \text { A8 } & 120.01134 \\ \text { A9 } & 120.008 \\ \text { A10 } & 120.01279 \\ \text { A11 } & 118.39342 \\ \text { A12 } & 118.39269 \\ \text { D1 } & -0.05684 \\ \text { D2 } & 0.03411 \\ \text { D3 } & 0.03235 \\ \text { D4 } & -179.97984 \\ \text { D5 } & 179.95325 \\ \text { D6 } & -179.99644 \\ \text { D7 } & -180 . \\ \text { D8 } & 179.98918 \\ \text { D9 } & 179.96185 \\ \text { D10 } & -90.01246 \\ \text { D11 } & 89.98748\end{array}$

$\mathrm{HF}=-436.7600811$

\section{Pyridine charge calculations:}

\#p opt b3lyp/6-31+g(d) geom=connectivity aim pop=npa

Symbolic Z-matrix:

Charge $=0$ Multiplicity $=1$

C

C $\quad 1 \quad$ B1

$\begin{array}{lllll}\mathrm{C} & 2 & \mathrm{~B} 2 & 1 & \mathrm{~A} 1\end{array}$

$\begin{array}{llllllll}\mathrm{C} & 3 & \mathrm{~B} 3 & 2 & \text { A2 } & 1 & \text { D1 } & 0\end{array}$

$\begin{array}{llllllll}\mathrm{C} & 4 & \mathrm{~B} 4 & 3 & \mathrm{~A} 3 & 2 & \mathrm{D} 2 & 0\end{array}$

$\begin{array}{llllllll}\mathrm{N} & 1 & \mathrm{~B} 5 & 2 & \mathrm{~A} 4 & 3 & \mathrm{D} 3 & 0\end{array}$

$\begin{array}{llllllll}\mathrm{H} & 3 & \mathrm{~B} 6 & 2 & \mathrm{~A} 5 & 1 & \mathrm{D} 4 & 0\end{array}$

$\begin{array}{llllllll}\mathrm{H} & 1 & \mathrm{~B} 7 & 6 & \mathrm{~A} 6 & 5 & \mathrm{D} 5 & 0\end{array}$

$\begin{array}{llllllll}\mathrm{H} & 2 & \mathrm{~B} 8 & 1 & \mathrm{~A} 7 & 6 & \mathrm{D} 6 & 0\end{array}$

$\begin{array}{llllllll}\mathrm{H} & 4 & \mathrm{~B} 9 & 3 & \mathrm{~A} 8 & 2 & \mathrm{D} 7 & 0\end{array}$ 


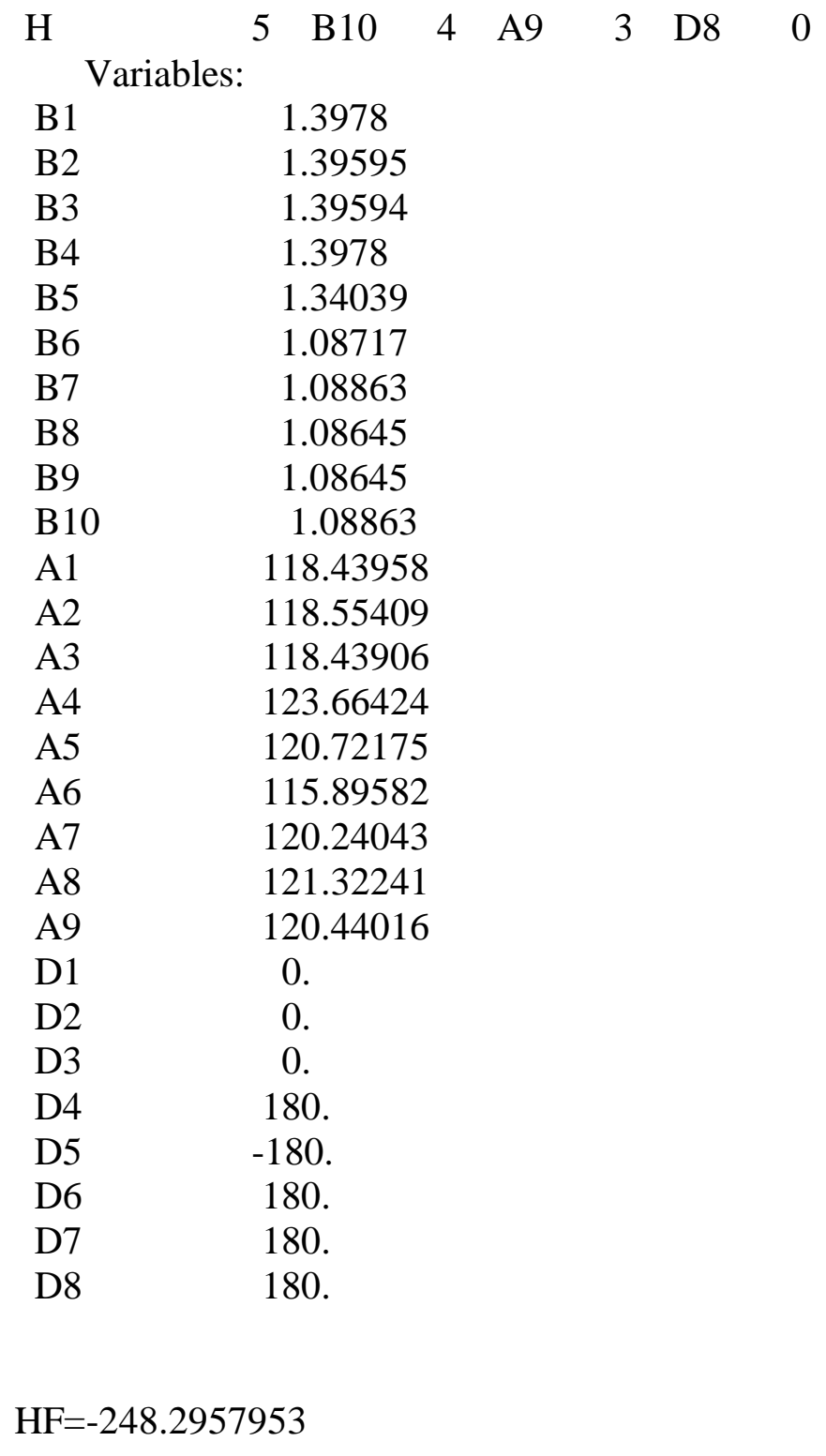




\section{Isonicotinic Acid:}

\#p b3lyp/aug-cc-pvdz opt freq guess=check geom=check symm=loose

Charge $=0$ Multiplicity $=1$

C, $0,-1.2328545993,0 .,-1.8574942094$

C, $0,-1.243354358,0 .,-0.4614178361$

C, $0,-0.0178169324,0 ., 0.2146493877$

C, $0,1.1614561763,0 .,-0.5397552065$

$\mathrm{C}, 0,1.0568516413,0 .,-1.9336409853$

$\mathrm{N}, 0,-0.1103138625,0 .,-2.591137476$

$\mathrm{H}, 0,-2.1725051081,0 .,-2.4141432825$

$\mathrm{H}, 0,-2.1769729875,0 ., 0.0984246528$

$\mathrm{H}, 0,2.1365748792,0 .,-0.0575615996$

$\mathrm{H}, 0,1.9580853741,0 .,-2.5508263549$

C, $0,-0.0200376193,0 ., 1.7084180295$

$\mathrm{O}, 0,1.2292054143,0 ., 2.2387804635$

O,0,-1.0191502457,0.,2.3954772779

H,0,1.1211076786,0.,3.2034519052

$\mathrm{HF}=-436.9209131$

\section{Isonicotinoate:}

Charge $=-1$ Multiplicity $=1$

C,0,0.,1.1413015511,-1.854649986

C,0,0., 1.1925301983,-0.4585590962

C, $0,0 ., 0 ., 0.2764726904$

C,0,0.,-1.1925301983,-0.4585590962

C,0,0.,-1.1413015511,-1.854649986

$\mathrm{N}, 0,0 ., 0 .,-2.5660990936$

H,0,0.,2.0669122165,-2.4401672677

$\mathrm{H}, 0,0 ., 2.1394058876,0.0814869936$

$\mathrm{H}, 0,0 .,-2.1394058876,0.0814869936$

$\mathrm{H}, 0,0 .,-2.0669122165,-2.4401672677$

C, $0,0 ., 0 ., 1.8287752511$

O,0,0.,-1.1384387341,2.3629422213

O,0,0.,1.1384387341,2.3629422213

$\mathrm{HF}=-436.3806922$ 


\section{Para Anion:}

\#p opt guess=check geom=check b3lyp/aug-cc-pVDZ symm=loose

Charge $=-1$ Multiplicity $=1$

C, $0,-1.1518679065,0.000000001,-0.6579500122$

$\mathrm{C}, 0,-1.1648317737,0.0000000003,0.7371760045$

C, $0,0.0178774493,-0.0000000008,1.5510313408$

C, $0,1.181513686,-0.0000000011,0.7101316303$

C, $0,1.1363966187,-0.0000000004,-0.6843249357$

$\mathrm{N}, 0,-0.0161690248,0.0000000007,-1.4028099816$

$\mathrm{H}, 0,-2.0893136429,0.0000000019,-1.2246872536$

$\mathrm{H}, 0,-2.1532243412,0.0000000007,1.2152225625$

H,0,2.1806622943,-0.0000000019,1.165269453

$\mathrm{H}, 0,2.0605304211,-0.0000000006,-1.2725190573$

$\mathrm{HF}=-247.6808053$

\#p opt mp2/aug-cc-pvdz symm=loose guess=check geom=check

Charge $=-1$ Multiplicity $=1$

C, $0,-1.1404815966,0.000000001,-0.6593765595$

C,0,-1.1574309787,0.0000000003,0.7410144943

C, $0,0.0177971761,-0.0000000008,1.5440669129$

C, $0,1.1742033316,-0.0000000011,0.7141396834$

C, $0,1.124980453,-0.0000000003,-0.6854886578$

$\mathrm{N}, 0,-0.0161433807,0.0000000007,-1.4005851208$

$\mathrm{H}, 0,-2.0831009485,0.0000000019,-1.2290245103$

$\mathrm{H}, 0,-2.1513462177,0.0000000007,1.2168036601$

$\mathrm{H}, 0,2.178821113,-0.0000000019,1.16689342$

H,0,2.0542194066,-0.0000000006,-1.2767119636

$\mathrm{HF}=-246.1281757$ 